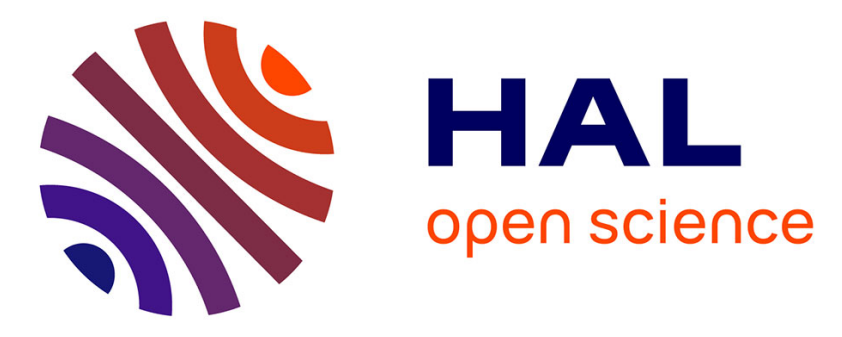

\title{
Multispectral Demosaicing Using Intensity in Edge-Sensing and Iterative Difference-Based Methods
}

Sofiane Mihoubi, Olivier Losson, Benjamin Mathon, Ludovic Macaire

\section{To cite this version:}

Sofiane Mihoubi, Olivier Losson, Benjamin Mathon, Ludovic Macaire. Multispectral Demosaicing Using Intensity in Edge-Sensing and Iterative Difference-Based Methods. 12th International IEEE Conference on Signal-Image Technology \& Internet-based Systems (SITIS 2016), Nov 2016, Naples, Italy. pp.805-810, 10.1109/SITIS.2016.132 . hal-01409705

\section{HAL Id: hal-01409705 https://hal.science/hal-01409705}

Submitted on 25 Jan 2018

HAL is a multi-disciplinary open access archive for the deposit and dissemination of scientific research documents, whether they are published or not. The documents may come from teaching and research institutions in France or abroad, or from public or private research centers.
L'archive ouverte pluridisciplinaire HAL, est destinée au dépôt et à la diffusion de documents scientifiques de niveau recherche, publiés ou non, émanant des établissements d'enseignement et de recherche français ou étrangers, des laboratoires publics ou privés. 


\title{
Multispectral Demosaicing Using Intensity in Edge-Sensing and Iterative Difference-Based Methods
}

\author{
Sofiane Mihoubi, Olivier Losson, Benjamin Mathon and Ludovic Macaire \\ CRIStAL - UMR 9189 \\ Université de Lille Sciences et Technologies, Bâtiment P2 \\ 59655 Villeneuve d'Ascq Cedex France \\ Email:sofiane.mihoubi@ed.univ-lille1.fr,olivier.losson@univ-lille1.fr, \\ benjamin.mathon@univ-lille1.fr,ludovic.macaire@univ-lille1.fr
}

\begin{abstract}
Single-sensor multispectral cameras, that sample spectral channels using a multispectral filter array, have recently emerged. They provide a raw image in which each channel is spectrally sampled pixel-wise according to the filter array pattern. A demosaicing procedure is then needed to estimate a multispectral image with full spectral resolution. The usefulness of intensity-based demosaicing has been shown in a previous work. In this paper, we both propose an intensity-based adaptation of the Binary Tree-based Edge-Sensing method for raw images with no dominant spectral band, and a new iterative method that uses the central wavelength distance between spectral bands.
\end{abstract}

\section{Keywords}

Multispectral demosaicing; intensity estimation; edge-sensitive method; spectral correlation; spatial correlation.

\section{INTRODUCTION}

In contrast with color imaging, digital multispectral images have higher spectral resolution in the visible and near infrared domains. These images usually reach from four to twenty spectral channels, each one typically matching with a specific and narrow spectral band. For nearly twenty years, multispectral images are acquired by selecting a different optical filter for each spectral channel [1].

Recently a single-sensor multispectral snapshot technology has emerged [2-4]. It uses a multispectral filter array (MSFA) to capture a single raw image. The MSFA is made of spectrally selective filters arranged in a periodic mosaic defined by a basic pattern. It provides a raw image in which each channel is spatially sampled according to the pattern. Each pixel in this raw image is characterized by the level of a single channel while the others have to be estimated to recover each channel in full definition. This process, known as demosaicing, is similar in its principle to the estimation of RGB components in single-sensor color cameras fitted with a Bayer color filter array (CFA) [5, 6]. Whereas CFA demosaicing is a well-studied problem for more than thirty years, MSFA demosaicing is a recent subject with new issues.

CFA demosaicing classically uses spatial correlation (correlation between spatially close pixels within a channel) and spectral correlation (correlation between levels of different channels at the same pixel). In multispectral demosaicing, the sparser spatial sampling of each channel weakens the assumption of spatial 


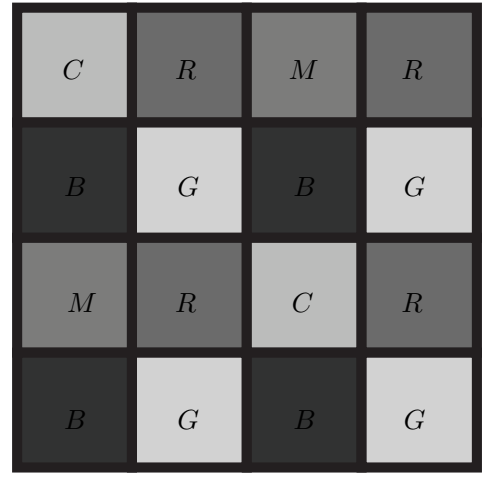

(a)

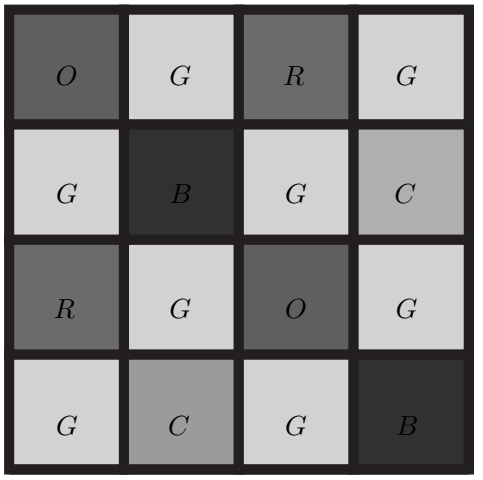

(b)

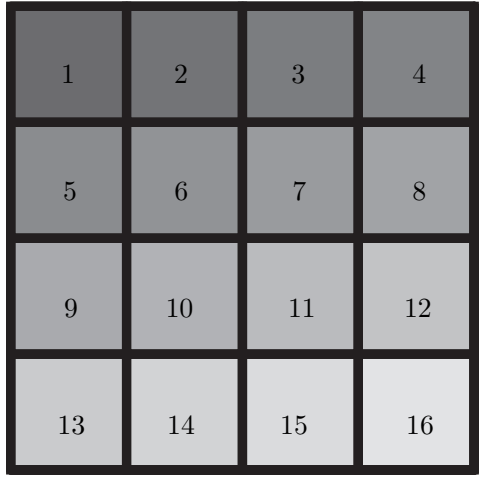

(c)

Figure 1. $4 \times 4$ basic MSFA patterns: (a) with three dominant bands [9], (b) with one dominant band [10], (c) with 16 different bands (the displayed numbers show the band indexes).

correlation. Moreover, the finer spectral sampling with narrow spectral bands implies that only spectrally close channels overlap. That weakens the assumption of spectral correlation between spectrally distant channels and introduces new properties: i) spectrally close channels are then more correlated than distant ones [7] and ii) all channels are strongly correlated with the intensity [8].

Two main contributions are presented in this paper: as the intensity channel carries edge information, we propose to integrate the intensity in an edge-sensing demosaicing scheme. Moreover, we improve the iterative difference-based method of [8] by computing the intensity with spectrally close channels.

This paper is organized as follows. First, we present the MSFAs proposed in the literature and the associated demosaicing methods, before we formulate the demosaicing problem for our retained MSFA pattern. Second, we recall the intensity estimation and present our edge sensing and iterative difference-based methods. Finally, we experimentally evaluate the proposed and state of art methods both objectively and with a few visual examples.

\section{MSFA DEMOSAICING}

\section{A. Multispectral filter arrays}

Most MSFAs are defined by a basic repetitive pattern and can be generated thanks to a binary tree [9]. Fig. 1 illustrates the kinds of basic patterns that are usually used in MSFA imaging:

- redundant patterns, that exhibit one or more dominant spectral band(s) (see Fig. 1(a)), or a Green $(G)$ dominant spectral band representing half of the samples (see Fig. 1(b));

- non-redundant patterns, that do not have any dominant spectral band and are usually square (see Fig. 1(c)).

In this article, we focus on the MSFA based on the pattern with $4 \times 4$ bands of Fig. 1(c) (hereafter qualified as non-redundant MSFA for short) for two main reasons. First, sampling all spectral bands only once in the basic pattern provides the most compact MSFAs, which allows us to consider a high number of channels (16) with limited estimation errors (as shown in the experimental section). Second, an MSFA with a square nonredundant pattern allows us to mimic IMEC's snapshot multispectral camera [3], that is—-to our knowledge—-the sole off-the-shelf MSFA-based system available on the market today. 


\section{B. MSFA demosaicing methods}

Among multispectral demosaicing methods, only Weighted Bilinear (WB) interpolation and Binary Treebased Edge-Sensing method (BTES) are adapted to any $4 \times 4$ MSFA. WB only uses spatial correlation by computing a weighted average of neighboring levels, while BTES estimates the non-dominant spectral channels by using edge-sensing weights computed on the estimated dominant ones (spectral correlation). Miao et al. [9] show that BTES gives better demosaicing performances than WB for redundant MSFAs. Note that these performances are similar for non-redundant MSFAs.

Monno et al. propose several methods specific to the basic pattern of Fig. 1(b): the original algorithm [10] uses an adaptive kernel up-sampling, which is improved in [11] by using a guided filter.

To demosaic raw images provided by non-redundant MSFAs, Wang et al. extend methods designed for the Bayer CFA to MSFA demosaicing. These methods use the discrete wavelet transform [12], median filtering [13], and a linear interpolation algorithm [14]. The first approach besides relies on a dominant green channel to estimate the luminance. Aggarwal and Majumdar [15] take the spatial (inter-pixel) correlation into account by learning the interpolation weights from a database. However, the performance of this approach highly depends on the learning database. Brauers and Aach [16] take the spectral (inter-channel) correlation into account in a demosaicing algorithm based on the spectral channel differences. Mizutani et al. [7] propose to iterate Brauers and Aach's scheme a number of times given by the spectral proximity of the channel pair involved in each difference, considering that the spectral correlation is stronger between spectrally close channels. We show [8] that the intensity of a multispectral image can be estimated from the raw MSFA image, assuming that the correlation between channels and the intensity is stronger than inter-channel correlation. The proposed method based on intensity differences (ID) [8] outperforms that based on spectral differences (SD) [16], which holds with iterative versions of these two algorithms (ItID [8] and ItSD [7]).

\section{MSFA Demosaicing problem formulation}

According to the MSFA pattern, each pixel $p$ of a raw MSFA image (denoted as $I^{M S F A}$ ) is associated with a single band $k \in\{1, \ldots, 16\}$. Let $S$ be the set of all pixels and $S^{k}$ be the pixel subset where the MSFA samples the band $k$, such that $S=\bigcup_{k=1}^{16} S^{k}$. Then, $I^{M S F A}$ can be seen as a spectrally-sampled version of the reference fully-defined image $\mathbf{I}=\left\{I^{k}\right\}_{k=1}^{16}$ according to the location of $p$ : for all $p \in S^{k}, I_{p}^{M S F A}=I_{p}^{k}$. At each pixel of $I^{M S F A}$, only one out of the 16 channels is available and the levels of the 15 others are missing. Demosaicing is then performed on $I^{M S F A}$ to obtain an estimated image $\hat{\mathbf{I}}$ with 16 fully-defined channels, among which 15 are estimated at each pixel $p$ :

$$
\forall p \in S^{k}, \hat{\mathbf{I}}_{p}=\left(\hat{I}_{p}^{1}, \ldots, \hat{I}_{p}^{k-1}, I_{p}^{k}, \hat{I}_{p}^{k+1}, \ldots, \hat{I}_{p}^{16}\right)
$$

where $\hat{I}_{p}^{l}, l \neq k$, is the estimated level of channel $I^{l}$ at $p$.

One of the simplest demosaicing schemes estimates the missing levels at each pixel thanks to a weighted average of neighboring levels. The WB interpolation can be implemented in two steps [16]:

- First, for each channel index $k$, it builds a sparse raw image $\tilde{I}^{k}$ that contains the available raw levels at pixels in $S^{k}$ and zero elsewhere. This can be formulated as:

$$
\tilde{I}^{k}=I^{M S F A} \odot m^{k},
$$


where $\odot$ denotes the element-wise product and $m^{k}$ is a binary mask defined at each pixel $p$ by:

$$
m_{p}^{k}= \begin{cases}1 & \text { if } p \in S^{k} \\ 0 & \text { otherwise }\end{cases}
$$

- Second, it interpolates each channel as:

$$
\hat{I}^{k}=\tilde{I}^{k} * H
$$

where $*$ is the convolution operator, and $H$ is a low-pass filter defined according to the $4 \times 4$ non-redundant MSFA pattern of Fig. 1(c):

$$
H=\frac{1}{16}\left[\begin{array}{ccccccc}
1 & 2 & 3 & 4 & 3 & 2 & 1 \\
2 & 4 & 6 & 8 & 6 & 4 & 2 \\
3 & 6 & 9 & 12 & 9 & 6 & 3 \\
4 & 8 & 12 & 16 & 12 & 8 & 4 \\
3 & 6 & 9 & 12 & 9 & 6 & 3 \\
2 & 4 & 6 & 8 & 6 & 4 & 2 \\
1 & 2 & 3 & 4 & 3 & 2 & 1
\end{array}\right] .
$$

\section{INTENSITY-BASED METHODS}

\section{A. Intensity estimation}

We define the intensity level $I_{p}^{M}$ at each pixel $p$ of a fully-defined multispectral image as the average level over all channels:

$$
I_{p}^{M}=\frac{1}{16} \sum_{k=1}^{16} I_{p}^{k} .
$$

In a previous work [8], we show that the intensity can be estimated from the MSFA raw image as:

$$
\hat{I}^{M}=I^{M S F A} * M
$$

where $M$ is a $5 \times 5$ normalized convolution filter:

$$
M=\frac{1}{64} \cdot\left[\begin{array}{lllll}
1 & 2 & 2 & 2 & 1 \\
2 & 4 & 4 & 4 & 2 \\
2 & 4 & 4 & 4 & 2 \\
2 & 4 & 4 & 4 & 2 \\
1 & 2 & 2 & 2 & 1
\end{array}\right] .
$$

This filter is designed to take each channel into account with the same contribution, and its size is that of the smallest odd-size window that includes each channel at least once in the raw image. The estimated intensity $\hat{I}^{M}$ is used to set up two demosaicing methods: intensity differences (ID) and iterative intensity differences (ItID).

Here, we propose to use the intensity to improve both BTES method for non-redundant MSFAs and ItID method by using the spectral distance between channels. 


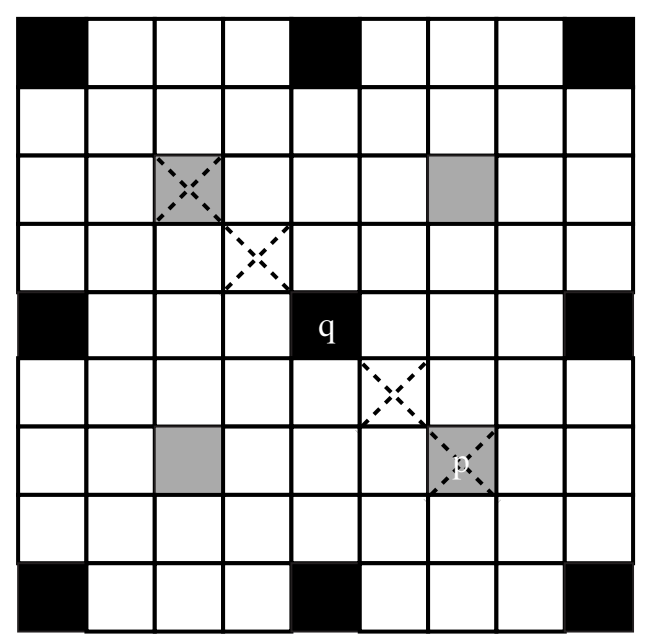

(a) $t=0$

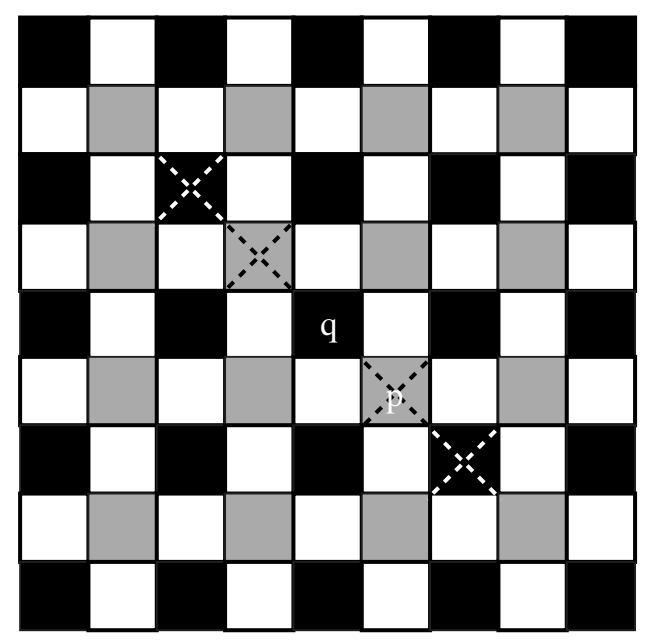

(c) $t=2$

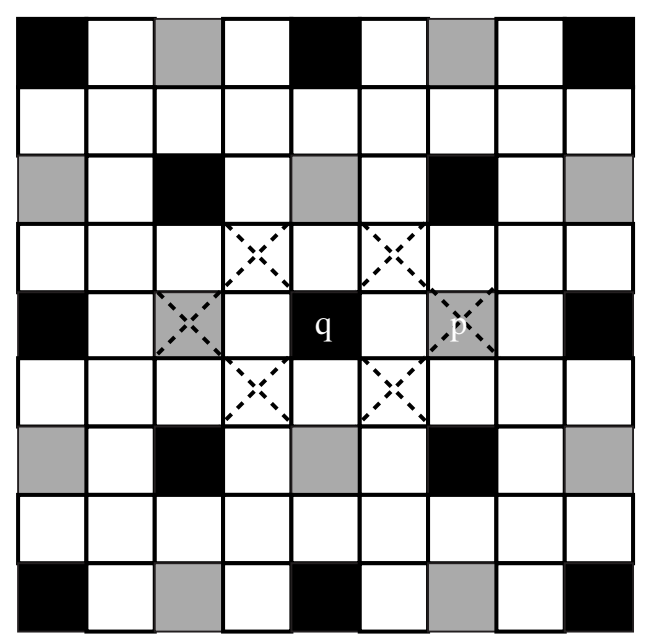

(b) $t=1$

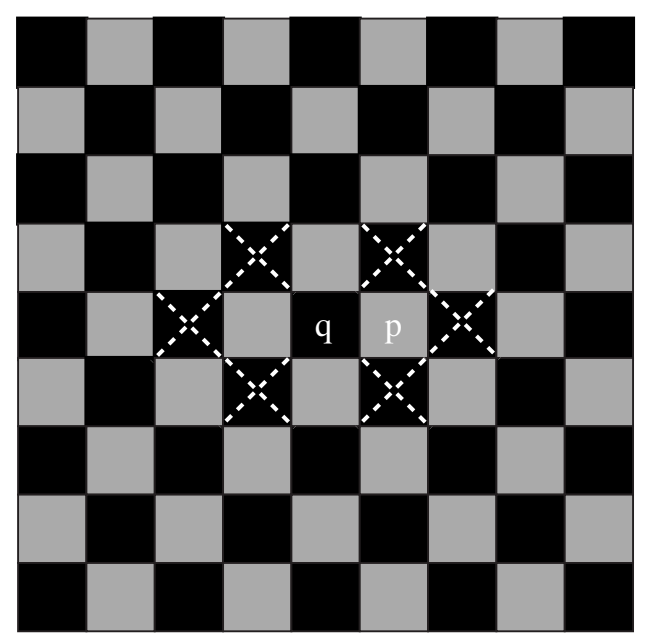

(d) $t=3$

Figure 2. Estimation of $\hat{I}^{k}$ in four steps by BTES method. At each step $t$, the subset of pixels whose levels are known or previously estimated are displayed in black. The subset of pixels whose levels are estimated at $t$ are displayed in gray. Considering a pixel $p$ to be estimated, the pixels used to compute the weight $\omega_{q}$ at neighbor $q$ are $q$ itself and those marked with a dotted cross.

\section{B. BTES for non-redundant MSFAs}

Miao et al. [9] propose a demosaicing scheme which, for a $4 \times 4$ non-redundant MSFA (see Fig. 1(c)), proceeds in the four successive steps shown in Fig. 2. For a given channel index $k$, at each step $t$, BTES only estimates the levels at the pixels displayed as gray in Fig. 2 from the known or previously estimated pixels (displayed as black). One of these levels is estimated at a pixel $p$ as:

$$
\hat{I}_{p}^{k}=\frac{\sum_{q \in N_{p}(t)} \omega_{q} \cdot \dot{I}_{q}^{k}}{\sum_{q \in N_{p}(t)} \omega_{q}},
$$

where $N_{p}(t)$ is the subset of the four closest neighbors $q$ of $p$ whose level at step $t$ (denoted as $\dot{I}_{q}^{k}$ ) is either available in $I^{M S F A}$ or has been previously estimated. The weights $\omega_{q}$ are set to 1 for $t \in\{0,1\}$, and are inversely proportional to a local gradient magnitude in the direction given by $p$ and $q$ for $t \in\{2,3\}$ [9]. 
Table I

EQUATIONS FOR $\omega_{q}$ IN THE PROPOSED IBTES METHOD, FOR THE TWO CONSIDERED DIRECTIONS AT EACH STEP $t \in[0,3]$.

DiRECTIONS: FIRST AND SECOND DiAGONAL (D1 AND D2), HORIZONTAL (H), AND VERTICAL (V).

\begin{tabular}{l|l|cc}
\hline$t$ & Dir. & \multicolumn{4}{|c}{ Equation for $\omega_{q}$} & \\
\hline \multirow{2}{*}{0} & $\mathrm{D} 1$ & $\left(1+\left|\hat{I}_{q+(2,2)}^{M}-\hat{I}_{q}^{M}\right|+\left|\hat{I}_{q+(-2,-2)}^{M}-\hat{I}_{q}^{M}\right|+\left|\hat{I}_{q+(-1,-1)}^{M}-\hat{I}_{q+(1,1)}^{M}\right|\right)^{-1}$ & $(10)$ \\
\cline { 2 - 5 } & $\mathrm{D} 2$ & $\left(1+\left|\hat{I}_{q+(2,-2)}^{M}-\hat{I}_{q}^{M}\right|+\left|\hat{I}_{q+(-2,2)}^{M}-\hat{I}_{q}^{M}\right|+\left|\hat{I}_{q+(-1,1)}^{M}-\hat{I}_{q+(1,-1)}^{M}\right|\right)^{-1}$ & $(11)$ \\
\hline \multirow{2}{*}{1} & $\mathrm{H}$ & $\left(1+\left|\hat{I}_{q+(2,0)}^{M}-\hat{I}_{q}^{M}\right|+\left|\hat{I}_{q+(-2,0)}^{M}-\hat{I}_{q}^{M}\right|+\frac{1}{2}\left|\hat{I}_{q+(-1,-1)}^{M}-\hat{I}_{q+(1,-1)}^{M}\right|+\frac{1}{2}\left|\hat{I}_{q+(-1,1)}^{M}-\hat{I}_{q+(1,1)}^{M}\right|\right)^{-1}$ & $(12)$ \\
\cline { 2 - 5 } & $\mathrm{V}$ & $\left(1+\left|\hat{I}_{q+(0,2)}^{M}-\hat{I}_{q}^{M}\right|+\left|\hat{I}_{q+(0,-2)}^{M}-\hat{I}_{q}^{M}\right|+\frac{1}{2}\left|\hat{I}_{q+(-1,-1)}^{M}-\hat{I}_{q+(-1,1)}^{M}\right|+\frac{1}{2}\left|\hat{I}_{q+(1,-1)}^{M}-\hat{I}_{q+(1,1)}^{M}\right|\right)^{-1}$ & $(13)$ \\
\hline \multirow{2}{*}{2} & $\mathrm{D} 1$ & $\left(1+\left|\dot{I}_{q+(2,2)}^{k}-\dot{I}_{q}^{k}\right|+\left|\dot{I}_{q+(-2,-2)}^{k}-\dot{I}_{q}^{k}\right|+\left|\hat{I}_{q+(-1,-1)}^{M}-\hat{I}_{q+(1,1)}^{M}\right|\right)^{-1}$ & $(14)$ \\
\cline { 2 - 5 } & $\mathrm{D} 2$ & $\left(1+\left|\dot{I}_{q+(2,-2)}^{k}-\dot{I}_{q}^{k}\right|+\left|\dot{I}_{q+(-2,2)}^{k}-\dot{I}_{q}^{k}\right|+\left|\hat{I}_{q+(-1,1)}^{M}-\hat{I}_{q+(1,-1)}^{M}\right|\right)^{-1}$ & $(15)$ \\
\hline \multirow{2}{*}{3} & $\mathrm{H}$ & $\left(1+\left|\dot{I}_{q+(2,0)}^{k}-\dot{I}_{q}^{k}\right|+\left|\dot{I}_{q+(-2,0)}^{k}-\dot{I}_{q}^{k}\right|+\frac{1}{2}\left|\dot{I}_{q+(-1,-1)}^{k}-\dot{I}_{q+(1,-1)}^{k}\right|+\frac{1}{2}\left|\dot{I}_{q+(-1,1)}^{k}-\dot{I}_{q+(1,1)}^{k}\right|\right)^{-1}$ & $(16)$ \\
\cline { 2 - 6 } & $\mathrm{V}$ & $\left(1+\left|\dot{I}_{q+(0,2)}^{k}-\dot{I}_{q}^{k}\right|+\left|\dot{I}_{q+(0,-2)}^{k}-\dot{I}_{q}^{k}\right|+\frac{1}{2}\left|\dot{I}_{q+(-1,-1)}^{k}-\dot{I}_{q+(-1,1)}^{k}\right|+\frac{1}{2}\left|\dot{I}_{q+(1,-1)}^{k}-\dot{I}_{q+(1,1)}^{k}\right|\right)^{-1}$ & $(17)$ \\
\hline
\end{tabular}

\section{Using Intensity in BTES (IBTES)}

To compute the weights $\omega_{q}$, Miao et al. take advantage of redundant MSFAs by using the dominant channel (e.g, $G$ in Fig. 1(b) that is estimated as shown in Fig. 2(d)). We follow the same strategy and use the intensity as a dominant channel. For this purpose we propose the weights of Tab. 1 that considers all the possible cases occurring with a $4 \times 4$ non-redundant MSFA. Fig. 2 shows the pixels used to compute these weights as dotted crosses on two examples: the first diagonal direction (for $t \in\{0,2\}$ ) and the horizontal direction (for $t \in\{1,3\}$ ). Among them, the pixels that do not overlie black (known) pixels at $t<3$ have unknown levels. We then replace them by the levels of the estimated intensity at the same position (see equations in Tab. 1). Note that each absolute difference involves two levels of the same channel. This intensity-adapted method is referred to as IBTES and assessed in Sec. IV.

\section{Iterative Intensity difference (ItID)}

The ID algorithm [8] consists in adding the estimated intensity $\hat{I}^{M}$ (see Eq. (7)) and the difference plane $\hat{\Delta}^{k}$ between any channel with index $k$ and the intensity:

$$
\hat{I}^{k}=\hat{I}^{M}+\hat{\Delta}^{k}
$$

where $\hat{\Delta}^{k}$ results from the bilinear interpolation (see Eq. (4)) of the sparse difference between MSFA raw data and the estimated intensity $\hat{I}^{M}$ at the pixels in $S^{k}$ :

$$
\hat{\Delta}^{k}=\left(I^{M S F A} \odot m^{k}-\hat{I}^{M} \odot m^{k}\right) * H .
$$

Once an initial fully-defined image $\hat{\mathbf{I}}(0)=\left\{\hat{I}^{k}(0)\right\}_{k=1}^{16}$ is estimated by ID (see Eq. (19)), ItID iteratively updates the intensity estimation using previously estimated channels as:

$$
\hat{I}^{M}(t)=\frac{1}{16} \cdot \sum_{k=1}^{16} \hat{I}^{k}(t-1)
$$


and then updates each channel by using the updated intensity $\hat{I}^{M}(t)$ as in Eqs. (19) and (20):

$$
\hat{I}^{k}(t)=\hat{I}^{M}(t)+\left(I^{M S F A} \odot m^{k}-\hat{I}^{M}(t) \odot m^{k}\right) * H .
$$

\section{E. Iterative Nearby Channel Difference (ItNCD)}

As shown in [7], spectrally close channels are more correlated than distant ones, and iterating using this property may improve the demosaicing performance. Here, we propose to iterate the ID algorithm by updating the intensity as an average over the spectrally closest channels rather than all channels like in ItID.

Specifically, we estimate the intensity in a different way for each channel by using its previously estimated level and those of the two closest channels. From the initial fully-defined image $\hat{\mathbf{I}}(0)$ estimated by ID, this new Iterative Nearby Channel Difference (ItNCD) algorithm uses two steps for each iteration $t>0$ :

- First, it computes 16 new images $\left\{\hat{I}^{M^{k}}(t)\right\}_{k=1}^{16}$ that estimate the intensity as:

$$
\hat{I}^{M^{k}}(t)= \begin{cases}\frac{\hat{I}^{1}(t-1)+2 \cdot \hat{I}^{2}(t-1)}{3} & \text { if } k=1, \\ \frac{2 \cdot \hat{I}^{15}(t-1)+\hat{I}^{16}(t-1)}{3} & \text { if } k=16 \\ \frac{\hat{I}^{k-1}(t-1)+\hat{I}^{k}(t-1)+\hat{I}^{k+1}(t-1)}{3} & \text { otherwise. }\end{cases}
$$

- Second, it computes each channel $\hat{I}^{k}(t)$ using the estimated intensity $\hat{I}^{M^{k}}(t)$ as:

$$
\hat{I}^{k}(t)=\hat{I}^{M^{k}}(t)+\left(I^{M S F A} \odot m^{k}-\hat{I}^{M^{k}}(t) \odot m^{k}\right) * H .
$$

Both ItID and ItNCD schemes are iterated a number $N$ of times (see Sec. IV-B) to provide the estimated multispectral image $\hat{\mathbf{I}}=\left\{\hat{I}^{k}(N)\right\}_{k=1}^{16}$.

\section{IMPLEMENTATION ON MULTISPECTRAL IMAGES}

\section{A. Reference and MSFA image simulation}

We perform the following experiments on the 32 multispectral images of size $512 \times 512$ pixels from the CAVE database [17]. Each image includes full spectral resolution reflectance data from $400 \mathrm{~nm}$ to $700 \mathrm{~nm}$ with a step of $10 \mathrm{~nm}$. To be consistent with a $4 \times 4$ non-redundant MSFA pattern, we select 16 channels such that the wavelength center of each channel ranges from 400 to $700 \mathrm{~nm}$ with a step of $20 \mathrm{~nm}$. These channels are rendered with the CIE D65 illuminant to get radiance data, then quantized on 8 bits. Finally, each reference image is a set of 16 radiance channels, where channel $I^{1}$ matches with the wavelength $400 \mathrm{~nm}$ and channel $I^{16}$ matches with the wavelength $700 \mathrm{~nm}$. The reference image is then spectrally sampled with the MSFA pattern of Fig. 1(c) to simulate a raw MSFA image.

The quality of an estimated image is evaluated by computing the average Peak Signal-to-Noise Ratio (PSNR) between the reference and estimated images as:

$$
\operatorname{PSNR}[\mathbf{I}, \hat{\mathbf{I}}]=\frac{1}{16} \cdot \sum_{k=1}^{16} 10 \cdot \log _{10}\left(\frac{255^{2}}{\frac{1}{|S|} \sum_{p \in S}\left(I_{p}^{k}-\hat{I}_{p}^{k}\right)^{2}}\right) .
$$

To avoid border effects, we only ignore 13 border pixels of both images in PSNR computation. 


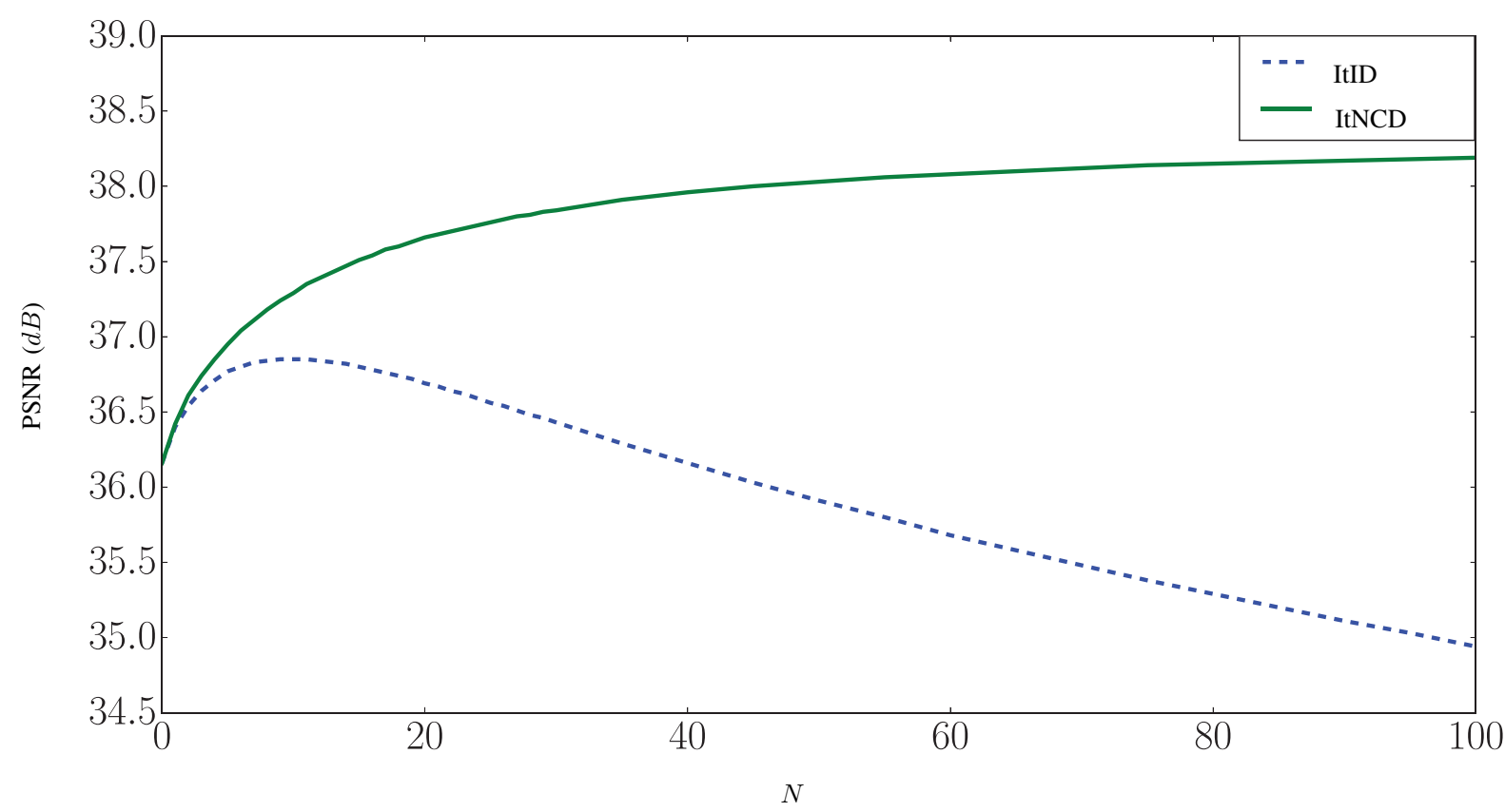

Figure 3. PSNR (average values over the 32 CAVE images) provided by ItID and ItNCD with respect to the number of iterations $N$.

\section{B. Number of iterations}

To determine the best number of iterations $N$, we assess the PSNR (averaged over the 32 images) reached by ItID and ItNCD methods with respect to $N$ when $N$ ranges from 0 to 100. The results (see Fig. 3) show that iterating ID first improves the demosaicing quality but then noticeably deteriorates it for more than 10 iterations, up to a deterioration of ID performances by $1.22 d B$ for 100 iterations. This is because ItID uses information from distant channels to compute the intensity, hence generating edge artifacts, that become prominent after 10 iterations. In contrast, using the intensity of nearby channels in ItNCD continuously improves ID performances, reaching an improvement of $2.03 \mathrm{~dB}$ for 100 iterations. Note that ItNCD always provides better PSNR than ItID. For the rest of our experiments, we set $N_{I t I D}=7$ and $N_{I t N C D}=20$ since ItID reaches its maximum value after 7 iterations and ItNCD reaches $75 \%(1.5 \mathrm{~dB})$ of its maximum improvement of ID after 20 iterations.

\section{Assessment of demosaicing methods}

We compare the performances reached by our proposed two methods (IBTES and ItNCD) with those provided by state-of-the-art methods for non-redundant MSFAs, namely the Weighted Bilinear (WB), Binary-Tree EdgeSensing (BTES [9]), Intensity Difference (ID [8]) and Iterative Intensity Difference (ItID [8]) methods.

Table II shows the PSNR values provided by the six methods for the 32 images of CAVE database, the highest PSNR for each row being highlighted in bold font. These results show that BTES provides similar PSNR than WB. Using intensity in IBTES improves the PSNR of BTES by $1.65 d B$ on average and at least by $0.67 \mathrm{~dB}$ on each image. IBTES also provides better results than ID for 15 out of the 32 images and gives similar average results than ID. Let us examine the performances reached by the two iterative methods. ItNCD provides the best result for all the images except one. Moreover, we can see that ItNCD improves the PSNR of ItID by $0.83 \mathrm{~dB}$ since this method uses the intensity of nearby channels instead of all channels. Finally, 
Table II

PSNR $(d B)$ PROVIDED BY THE DEMOSAICING METHODS (WB [16], BTES [9], ID [8], ItID [8], AND OUR TWO PROPOSED METHODS IBTES AND ItNCD) ON THE 32 CAVE IMAGES. FAR STANDS FOR "FAKE AND REAL" AND RAF FOR "REAL AND FAKE".

\begin{tabular}{|c|c|c|c|c|c|c|}
\hline Image Method & WB & BTES & IBTES & ID & ItID & ItNCD \\
\hline Balloons & 40.04 & 40.24 & 42.90 & 41.82 & 42.52 & 43.20 \\
\hline Beads & 26.77 & 26.89 & 27.56 & 28.01 & 28.10 & 29.18 \\
\hline CD & 37.38 & 37.58 & 38.52 & 37.94 & 37.65 & 37.89 \\
\hline Chart and stuffed toy & 29.62 & 29.68 & 31.26 & 30.89 & 31.82 & 32.13 \\
\hline Clay & 39.07 & 39.24 & 41.39 & 40.27 & 40.41 & 42.93 \\
\hline Cloth & 26.86 & 26.87 & 27.63 & 28.38 & 29.29 & 29.36 \\
\hline Egyptian statue & 37.66 & 37.70 & 39.08 & 39.32 & 40.36 & 40.56 \\
\hline Face & 38.69 & 38.79 & 41.12 & 40.29 & 41.04 & 41.26 \\
\hline FaR beers & 37.57 & 37.72 & 39.23 & 39.41 & 39.57 & 40.58 \\
\hline FaR food & 37.00 & 37.16 & 38.40 & 38.57 & 38.63 & 39.84 \\
\hline FaR lemon slices & 37.64 & 37.77 & 40.30 & 39.39 & 40.53 & 40.77 \\
\hline FaR lemons & 33.84 & 34.00 & 35.40 & 35.22 & 35.79 & 36.10 \\
\hline FaR peppers & 36.77 & 36.89 & 38.81 & 39.55 & 40.16 & 41.54 \\
\hline FaR strawberries & 35.55 & 35.64 & 37.32 & 37.34 & 38.54 & 38.54 \\
\hline FaR sushi & 36.15 & 36.24 & 37.30 & 38.03 & 38.49 & 39.22 \\
\hline FaR tomatoes & 34.33 & 34.42 & 35.28 & 35.72 & 36.41 & 36.74 \\
\hline Feathers & 31.21 & 31.28 & 32.98 & 33.17 & 34.40 & 35.10 \\
\hline Flowers & 36.03 & 36.15 & 37.87 & 38.03 & 38.42 & 39.66 \\
\hline Glass tiles & 27.17 & 27.21 & 27.94 & 28.66 & 29.66 & 30.84 \\
\hline Hairs & 34.07 & 34.16 & 36.06 & 35.74 & 36.84 & 37.11 \\
\hline Jelly beans & 28.38 & 28.52 & 29.66 & 30.54 & 31.71 & 32.27 \\
\hline Oil painting & 30.78 & 30.79 & 31.65 & 30.96 & 32.02 & 33.59 \\
\hline Paints & 29.79 & 30.02 & 32.00 & 32.61 & 32.91 & 34.92 \\
\hline Photo and face & 35.99 & 36.10 & 38.63 & 38.28 & 39.59 & 39.96 \\
\hline Pompoms & 38.25 & 38.49 & 39.96 & 39.08 & 38.66 & 40.11 \\
\hline RaF apples & 39.88 & 40.11 & 43.26 & 41.97 & 42.78 & 43.34 \\
\hline RaF peppers & 36.73 & 36.87 & 39.57 & 38.53 & 39.71 & 39.97 \\
\hline Sponges & 35.41 & 35.61 & 37.57 & 36.83 & 36.72 & 39.05 \\
\hline Stuffed toys & 35.63 & 35.87 & 38.86 & 38.20 & 38.11 & 39.84 \\
\hline Superballs & 37.69 & 37.80 & 38.70 & 39.47 & 40.22 & 40.72 \\
\hline Thread spools & 31.84 & 31.91 & 32.80 & 33.97 & 35.55 & 36.84 \\
\hline Watercolors & 28.82 & 28.88 & 30.27 & 30.96 & 31.84 & 31.91 \\
\hline Average & 34.46 & 34.58 & 36.23 & 36.16 & 36.83 & 37.66 \\
\hline
\end{tabular}



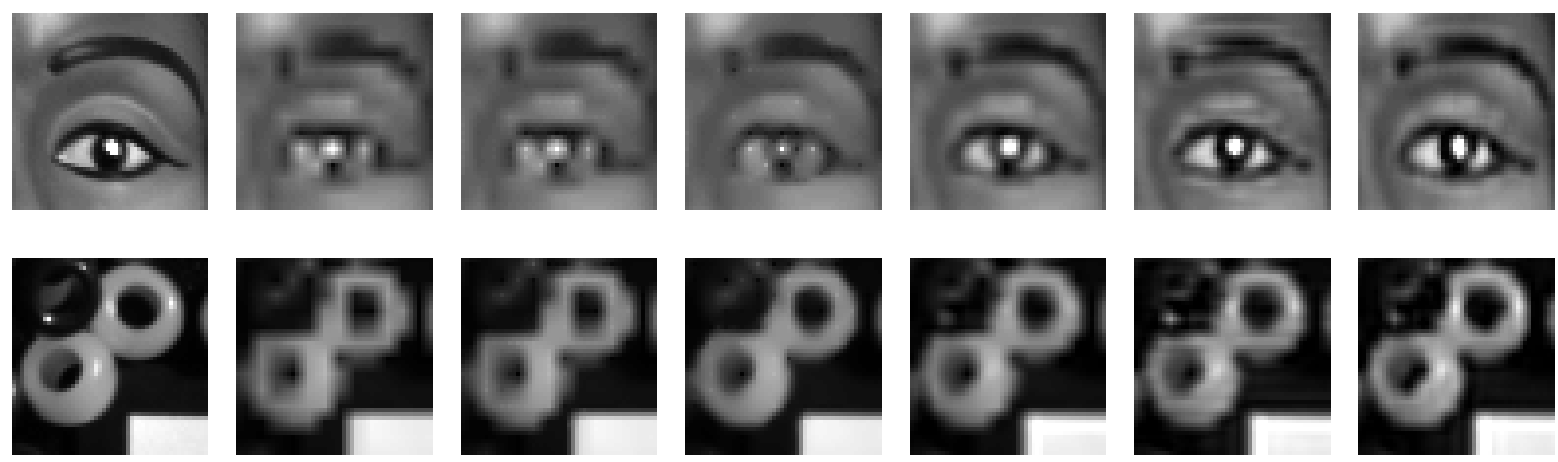

(a) Reference

(b) WB

(c) BTES

(d) IBTES
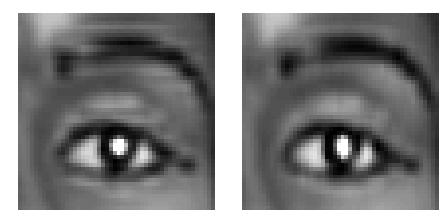

Figure 4. Central area of the reference channel $I^{9}(560 \mathrm{~nm})$ (a) and estimated channel $\hat{I}^{9}$ (b)-(g) of CAVE "Egyptian statue" (first row) and "Beads" (second row) multispectral images under CIE D65 illuminant.

iterating ID with ItNCD provides an improvement of $1.50 \mathrm{~dB}$.

To visually compare the results of the six methods, we have selected a central area of channel $I^{9}$ (at central wavelength $560 \mathrm{~nm}$ ) of the "Egyptian statue" and "Beads" CAVE images (see Fig. 4). The images estimated by WB and BTES are strongly blurred and have severe edge distortions. By using edge information of the intensity in IBTES, these artifacts are fairly reduced. The ID method fairly restores the sharpness of the reference image at the cost of visual artifacts. This sharpness but also the artifacts are accentuated with ItID, whereas ItNCD improves the sharpness effectively without generating so many artifacts.

\section{CONCLUSion}

This paper presents two main contributions to multispectral demosaicing. First, we adapt BTES scheme (as IBTES) for non-redundant MSFAs considering that the intensity is a dominant spectral channel. Instead of using bilinear interpolation on each channel, IBTES uses edge-sensitive weights computed on the intensity estimated from the raw MSFA image. Experimental results show that IBTES improves BTES for our $4 \times 4$ MSFA and provides estimated images with sharpest edges. Second, by computing a specific intensity defined as the average of close channels for each channel, we propose ItNCD that objectively improves ItID and visually generates fewer artifacts. Future work will focus on how to still improve the intensity estimation for intensity-based demosaicing and on experiments with real data and sensor noise consideration.

\section{ACKNOWLEDGMENT}

This work has been performed thanks to the ANR-11-EQPX-23 IrDIVE platform.

\section{REFERENCES}

[1] J. Qin, K. Chao, M. S. Kim, R. Lu, and T. F. Burks, "Hyperspectral and multispectral imaging for evaluating food safety and quality," Journal of Food Engineering, vol. 118, no. 2, pp. 157-171, 2013.

[2] J.-B. Thomas, P.-J. Lapray, P. Gouton, and C. Clerc, "Spectral characterization of a prototype SFA camera for joint visible and NIR acquisition," Sensors, vol. 16, no. 7, p. 993, 2016. 
[3] B. Geelen, N. Tack, and A. Lambrechts, “A compact snapshot multispectral imager with a monolithically integrated per-pixel filter mosaic," in Procs. SPIE: Advanced Fabrication Technologies for Micro/Nano Optics and Photonics VII, vol. 8974, San Francisco, CA, USA, Feb. 2014, pp. 89740L-89740L-8.

[4] Y. Monno, S. Kikuchi, M. Tanaka, , and M. Okutomi, “A practical one-shot multispectral imaging system using a single image sensor," IEEE Trans. Image Process., vol. 24, no. 10, pp. 3048-3059, Oct. 2015.

[5] I. Pekkucuksen and Y. Altunbasak, "Edge strength filter based color filter array interpolation," IEEE Trans. Image Process., vol. 21, no. 1, pp. 393-397, Jan 2012.

[6] K. Hirakawa and T. W. Parks, “Adaptive homogeneity-directed demosaicing algorithm,” IEEE Trans. Image Process., vol. 14, no. 3, pp. 360-369, March 2005.

[7] J. Mizutani, S. Ogawa, K. Shinoda, M. Hasegawa, and S. Kato, "Multispectral demosaicking algorithm based on inter-channel correlation," in Procs. IEEE Visual Communications and Image Processing Conf. (VCIP 2014), Valletta, Malta, Dec. 2014, pp. 474-477.

[8] S. Mihoubi, O. Losson, B. Mathon, and L. Macaire, "Multispectral demosaicing using intensity-based spectral correlation," in Procs. 5th Int. Conf. on Image Processing Theory, Tools and Applications (IPTA'15), Orléans, France, Nov. 2015, pp. 461-466.

[9] L. Miao, H. Qi, R. Ramanath, and W. Snyder, "Binary tree-based generic demosaicking algorithm for multispectral filter arrays," IEEE Trans. Image Process., vol. 15, no. 11, pp. 3550-3558, Nov. 2006.

[10] Y. Monno, M. Tanaka, and M. Okutomi, "Multispectral demosaicking using adaptive kernel upsampling," in Procs. 18th IEEE Int. Conf. on Image Processing (ICIP 2011), Brussels, Belgium, Sep. 2011, pp. 3157-3160.

[11] — - "Multispectral demosaicking using guided filter," in Procs. SPIE: Digital Photography VII, N. Sampat and S. Battiato, Eds., vol. 8299, Burlingame, CA, USA, Jan. 2012, pp. 82 990O-82990O7.

[12] X. Wang, J.-B. Thomas, J. Y. Hardeberg, and P. Gouton, "Discrete wavelet transform based multispectral filter array demosaicking," in Procs. 2013 Colour and Visual Computing Symposium (CVCS 2013), Gjøvik, Norway, Sep. 2013, pp. 1-6.

[13] — , "Median filtering in multispectral filter array demosaicking," in Procs. SPIE: Digital Photography IX, N. Sampat and S. Battiato, Eds., vol. 8660, Burlingame, CA, USA, Feb. 2013, pp. 86 600E-86 600E-10.

[14] C. Wang, X. Wang, and J. Y. Hardeberg, "A linear interpolation algorithm for spectral filter array demosaicking," in Procs. 6th EURASIP Int. Conf. on Image and Signal Processing (ICISP'2014), ser. LNCS, A. Elmoataz, O. Lezoray, F. Nouboud, and D. Mammass, Eds., vol. 8509. Cherbourg, France: Springer-Verlag Berlin, Jun. 2014, pp. 151-160.

[15] H. K. Aggarwal and A. Majumdar, "Single-sensor multi-spectral image demosaicing algorithm using learned interpolation weights," in Procs. 2014 Int. Geoscience and Remote Sensing Symposium (IGARSS 2014), Québec City, Québec, Canada, Jul. 2014, pp. 2011-2014.

[16] J. Brauers and T. Aach, "A color filter array based multispectral camera," in Institute of Imaging and Computer Vision, RWTH Aachen University, 52056 Aachen, Germany, Oct. 2006.

[17] F. Yasuma, T. Mitsunaga, D. Iso, and S. K. Nayar, "Generalized assorted pixel camera: Postcapture control of resolution, dynamic range, and spectrum," IEEE Trans. Image Process., vol. 19, no. 9, pp. 2241-2253, 
Sep. 2010. 